\title{
ASSESSMENT OF AN APPROACH TO GENERATING INFLOW SYNTHETIC TURBULENCE FOR LARGE EDDY SIMULATIONS OF COMPLEX TURBULENT FLOWS
}

\section{Y. Adamian and A. K. Travin}

St. Petersburg State Polytechnic University St. Petersburg, Russia

An approach to generating inflow synthetic turbulence recently developed by the authors has been applied to zonal Reynolds-Averaged Navier-Stokes (RANS) / Large Eddy Simulations (LES) of two complex turbulent flows: flow over a wall-mounted hump and hydrofoil trailing edge flow, and to a LES of a flow in a three-dimensional (3D) diffuser. The results show that the zonal RANS-LES approach with synthetic turbulence at the interface is in excellent agreement with experimental data for hump and trailing edge flows. For the diffuser flow, it is shown that results depend significantly on the RANS model used to provide averaged velocity and Reynolds stresses at the inlet.

\section{INTRODUCTION}

Large eddy simulation of spatially developing turbulent flows requires specification of unsteady (with a "turbulent content") velocity fields at inlet boundaries. For nearly self-similar flows, such fields can be created with the use of the socalled recycling techniques (see, e.g., [1]). However, for more complex flows, applicability of the recycling methods, even improved ones (see, e.g., [2]), becomes questionable, and other approaches should be used. In a recent paper of the authors [3], a simple synthetic turbulence generator has been proposed and, based on the simulations of a set of canonical shear flows (developed twodimensional (2D) channel flow, flat plate boundary layer, free shear layer) shown to be superior over similar methods available in literature [4-6] thanks to a capability of creating turbulent structures rapidly transforming to real turbulence downstream of the inlet boundaries. An objective of the present study is a more extensive validation of the method in the framework of zonal RANS-LES computations of complex turbulent flows. These include an aerodynamic flow with 
pressure induced separation and reattachment (the wall-mounted hump studied in the experiments [7] and used as a test case in many validation studies, e. g., [8]) and a hydrofoil trailing edge flow with shallow separation investigated in the experiment [9] and used for validation of different hybrid RANS-LES approaches in the EU project DESider [10].

One more validation test of the inflow generation method has been done, namely, the flow in a 3D diffuser studied in the experiments [11]. This flow is difficult to simulate by means of RANS turbulence models because of the presence of secondary flows driven by normal Reynolds stresses anisotropy. Since synthetic turbulence is usually generated according to a RANS solution, this can significantly worsen the LES solution. The diffuser flow using synthetic turbulence generated with $k-\omega$ shear stress transport (SST) [12] RANS and EARSM-WJ-BSL (Explicit Algebraic Reynolds Stress Model-Wallin-Johanson-Baseline) [13] RANS fields taken as the input has been simulated.

The rest of the paper is organized as follows: section 2 outlines the synthetic turbulence generation method, section 3 briefly describes turbulence models and numerical methods used in the simulations, sections 4-6 present simulation results for the wall-mounted hump flow, hydrofoil trailing edge flow and $3 \mathrm{D}$ diffuser flow, respectively, and, finally, section 7 contains conclusions of the study.

\section{SYNTHETIC TURBULENCE GENERATION METHOD}

The method has been described in detail in a recent paper by the authors [3]. Below follows a brief outline of the method highlighting only its main features.

The velocity field at the LES inflow is defined as a sum of steady RANS velocity field and synthetic field of velocity fluctuations multiplied by Cholesky decomposition of the Reynolds stress tensor:

$$
\mathbf{u}(\mathbf{r}, t)=\mathbf{U}_{\mathrm{RANS}}(\mathbf{r})+\mathbf{A} \mathbf{u}^{\prime}(\mathbf{r}, t) ; \quad \mathbf{R}=\mathbf{A}^{\mathrm{T}} \mathbf{A} .
$$

The velocity fluctuations field is prescribed in the form of weighted superposition of Fourier modes:

$$
\mathbf{u}^{\prime}(\mathbf{r}, t)=\sqrt{6} \sum_{n=1}^{N} \sqrt{q^{n}}\left[\sigma^{n} \cos \left(k^{n} \mathbf{d}^{n} \cdot \mathbf{r}+\varphi^{n}+s^{n} \frac{t}{\tau}\right)\right]
$$

where wavenumbers $k$ form geometric series, mode weights $q$ are calculated using the local energy spectrum (see below), $\tau$ is the global time scale, $\sigma, d, \varphi$, and $s$ are the random parameters: velocity direction of the mode, wave vector direction, phase, and time frequency (for details, see [3]). 
The weights of the modes are defined with the use of a modified von Karman spectrum:

$$
q^{n}=\frac{E\left(k^{n}\right) \Delta k^{n}}{\sum_{n=1}^{N} E\left(k^{n}\right) \Delta k^{n}} ; \quad E(k)=\left(\frac{k}{k_{e}}\right)^{4}\left[1+2.4\left(\frac{k}{k_{e}}\right)^{2}\right]^{-17 / 6} f_{\eta} f_{\text {cut }}
$$

where $f_{\eta}$ and $f_{\text {cut }}$ damp the spectrum near wavenumber corresponding to the Kolmogorov length-scale and the maximum resolvable wavenumber on the grid, wavenumber $k_{e}$ corresponding to the size of the most energy-carrying eddies is defined by the length scale $l_{e}$. The length scale is defined as follows:

$$
l_{e}=\min \left(2 d_{w}, C_{l} l_{t}\right)
$$

where $C_{l}=3$ is the empirical constant and $l_{t}$ is the length scale of the turbulence model used in RANS region (for $k-\omega$ model, $l_{t}=k_{t}^{1 / 2} /\left(C_{\mu} \omega_{t}\right)$ ).

The global time scale $\tau$ is defined by the maximum value of the length scale and a macroscale of the velocity at the LES inlet:

$$
\tau=\frac{C_{\tau} l_{e}^{\max }}{U}
$$

where $C_{\tau}=2$ is the empirical constant. Such global definition of the time scale coupled with the local scale of the most energy-carrying eddies (1) results in forming of physically realistic elongated in the streamwise direction eddies near the wall and nearly isotropic eddies away from the wall.

The method has shown to produce quality inflow turbulent content and ensure a rapid formation of realistic turbulent structures downstream of the inflow for canonical turbulent shear flows: plane channel flow, boundary layer flow, and mixing layer flow [3]. It has been shown that for wall-bounded flows, the synthetic turbulence needed relaxation region of about 2 boundary layer thickness lengths and did not worsen the wall friction significantly.

\section{TURBULENCE MODELS AND NUMERICAL METHODS}

For RANS simulations, $k-\omega$ SST model [12] has been used for all the flows and EARSM-WJ-BSL [13] model for the 3D diffuser flows. For LES and hybrid RANS-LES simulations, the Improved Delayed Detached Eddy Simulation (IDDES) [14] has been used. This model is solution-dependent and functions as wall-modeling LES model if the turbulent content is present in the solution 
and as a RANS model in attached boundary layer without resolved turbulent fluctuations.

For all the simulations, NTS finite-volume multiblock structured code [15] with overlapping grids capability has been used. The ability to use overlapping grids is crucial to simultaneous combined RANS-LES simulation using synthetic turbulence at the RANS-LES interface. The NTS code uses the method of Rogers and Kwak [16] for incompressible flows. Convective fluxes are computed with the use of the 4th order central-differencing scheme for LES and 3rd order upwind scheme for RANS. For diffusive fluxes, the code uses 2nd order central differencing scheme. Time integration is done using implicit 3-step 2nd order scheme with subiterations.

\section{WALL-MOUNTED HUMP FLOW}

The flow over a 2D wall-mounted hump has been studied in the experiments [7] and widely used as a validation test for turbulence modeling approaches [8]. Scheme of the flow is shown in Fig. 1.

The Reynolds number based on maximum inlet velocity and chord length is 936,000 . Upper wall is slippery and slightly adjusted to account for partial blockage effect as recommended in [8]. The computational domain extends from $x / c=-2.14$ to 4.0 . Velocity and turbulence variables profiles at the inlet plane have been obtained in a separate RANS calculation of zero-pressure gradient boundary layer at the Reynolds number based on momentum thickness equal to $\operatorname{Re}_{\theta}=7500$. The computational grid in $x-y$ plane has dimensions $375 \times 111$ and is nearly isotropic in the separation zone with $\Delta x / c \approx \Delta y / c \approx 5 \cdot 10^{-3}$. For hybrid and zonal RANS-LES simulations, the grid has 101 points with equal spacing $\Delta z / c=4 \cdot 10^{-3}$ in $z$ direction amounting to spanwise width of $L_{z} / c=0.4$. Periodic boundary conditions have been used in $z$ direction.

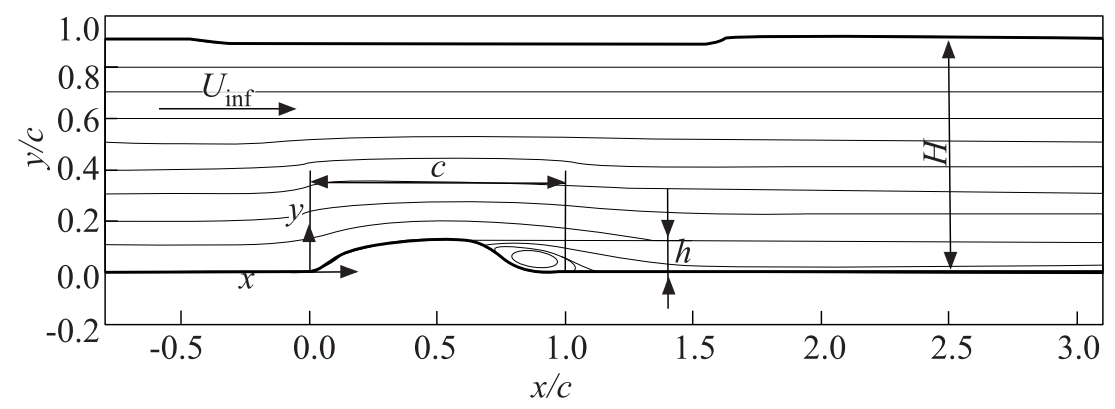

Figure 1 Schematic of the wall-mounted hump flow 


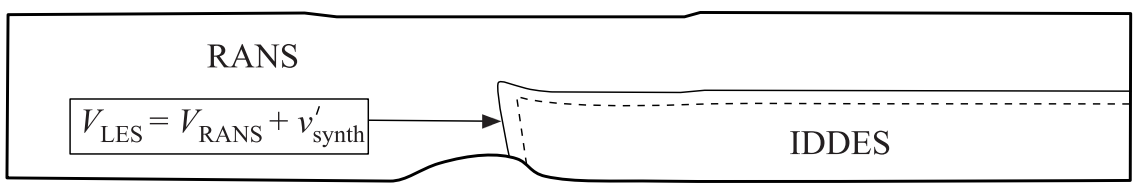

Figure 2 Layout of zonal RANS-IDDES simulation of wall-mounted hump flow

Three types of simulation have been done for this flow: 2D RANS using $k-\omega$ SST model, hybrid RANS-LES using IDDES method in the whole domain, and zonal RANS-LES using synthetic turbulence at the interface. For zonal RANS-LES simulation, the LES inlet plane was at near top of the hump. The RANS outlet was located somewhat farther downstream (20 grid points) to avoid contamination of RANS solution with resolved turbulent fluctuations (Fig. 2). Synthetic velocity field was prescribed at LES inlet, while at RANS outlet, the velocity and pressure were taken directly from the LES domain.

Some results of the simulations are shown in Figs. 3 and 4. Isosurfaces of $\lambda_{2}$ criterion showing resolved turbulent fluctuations are presented in Fig. 3. It can be seen from this figure that when IDDES is used in the whole domain, the separated boundary layer contains only unphysical large almost $2 \mathrm{D}$ vortices in the vicinity of separation point. This is typical for hybrid RANS-LES methods when the boundary layer does not contain resolved turbulent content before separation point. Zonal RANS-IDDES simulation is free from this drawback. Such a difference in structure of resolved turbulent fluctuations fields manifests itself also in different prediction of wall friction in the separation zone shown in Fig. 4. Zonal RANS-IDDES simulation provides correct level of wall friction in the whole separation zone while for IDDES in the whole domain, it is noticeably overpredicted at $0.8<x / c<1.0$. The RANS simulation using $k-\omega$ SST model severely overpredicts the length of the separation zone.

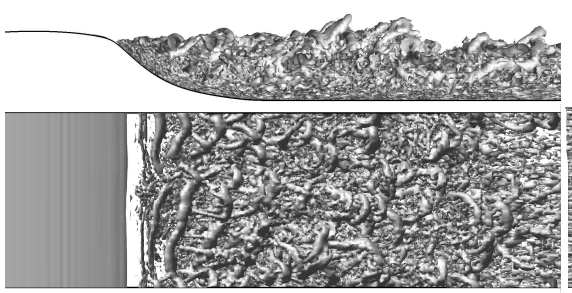

(a)

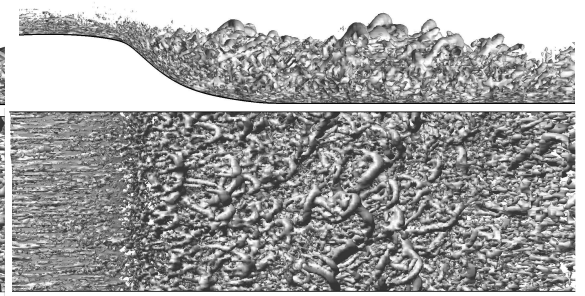

(b)

Figure 3 The $\lambda_{2}$ isosurfaces for wall-mounted hump flow: (a) IDDES in the whole domain; and (b) zonal RANS-IDDES 


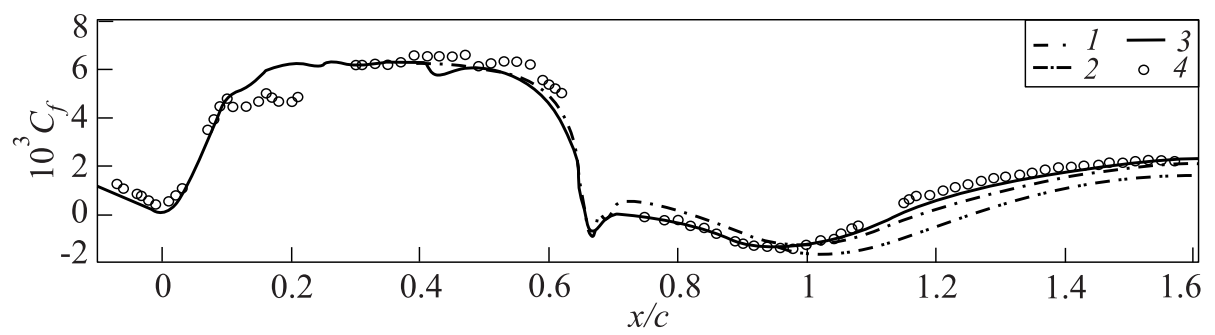

Figure 4 Wall friction comparison for the wall-mounted hump flow: $1-k-\omega$ SST RANS; $2-k-\omega$ SST IDDES; 3 - zonal RANS-IDDES; and 4 - experiments [7]

\section{HYDROFOIL TRAILING EDGE FLOW}

The scheme of the trailing edge flow is shown in Fig. 5. The Reynolds number based on hydrofoil thickness $h$ and freestream velocity $U_{\infty}$ is equal to $\operatorname{Re}_{h}=10^{5}$ according to the experiments [9].

Layout of the zonal RANS-LES simulation is shown in Fig. 5. The LES zone covers only the trailing edge and near wake, the rest is simulated by RANS using
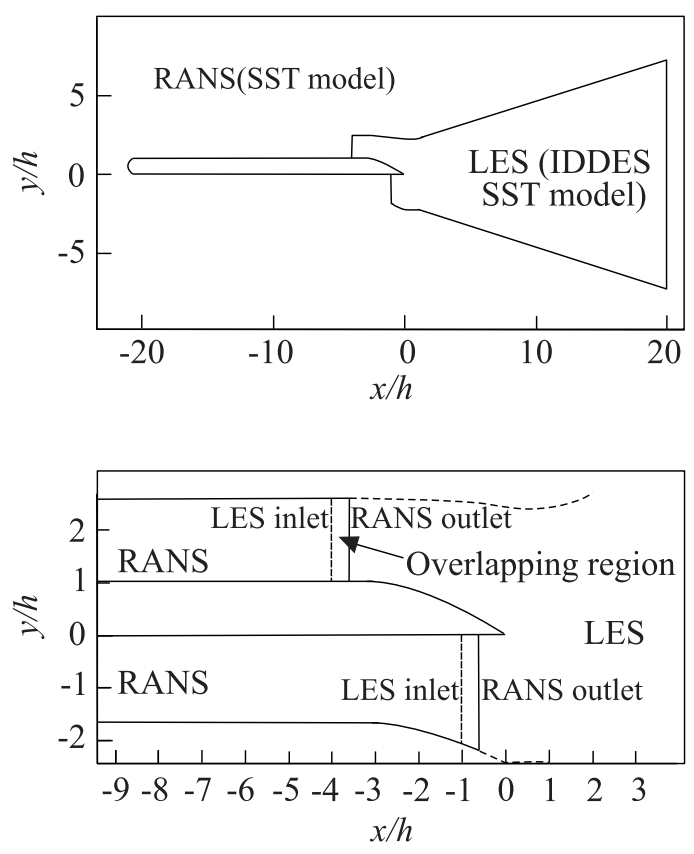

Figure 5 Layout of zonal RANS-LES simulation of hydrofoil trailing edge flow 


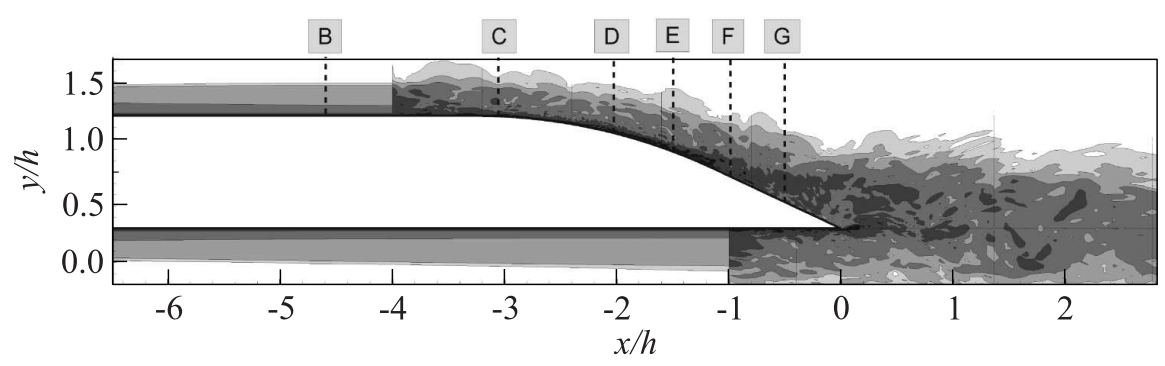

Figure 6 Vorticity magnitude field for zonal RANS-LES simulation of hydrofoil trailing edge flow: $\mathrm{B}-\mathrm{G}$ denote planes used to compare the profiles of the averaged velocity
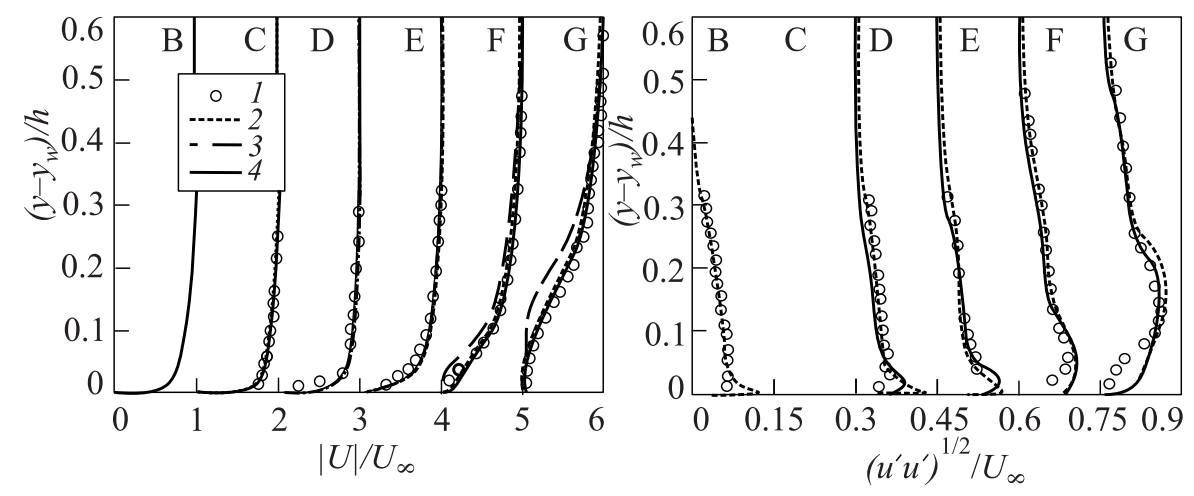

Figure 7 Comparison of averaged streamwise velocity and rms of streamwise velocity fluctuations profiles for hydrofoil trailing edge flow $\left(y_{w}\right.$ denotes $y$-coordinate of the wall): 1 - experiment; 2 - LES [17]; 3 - SST RANS; and 4 - zonal RANS-LES

$k-\omega$ SST model. The RANS and LES zones overlap for 20 grid points to make possible simultaneous RANS and LES simulations.

In the LES zone near the trailing edge, the grid is close to isotropic with spacing $\Delta x / h \approx \Delta y / h=0.02$. In $z$ direction, the grid has 101 points evenly spaced by $\Delta z / h=0.01$, so that spanwise width is $L_{z}=h$.

Freestream conditions $u=U_{\infty}, v=0$ have been used at the inlet boundary which is located at $x / h=-50$. Constant pressure boundary conditions have been used at the outlet boundary at $x / h=20$. In the $z$ direction, periodic boundary conditions have been used.

Some results of the zonal RANS-LES simulation are shown in Figs. 6 and 7. Figure 6 shows instant fields of vorticity magnitude in the $x-y$ plane demonstrating resolved turbulent content in the LES zone. Comparison of streamwise 
velocity profiles at selected locations (see Fig. 7) shows excellent agreement both with resolved LES using recycling methods [17] and experimental data [9]. The profiles of root mean squared (rms) streamwise velocity fluctuations also show good agreement with resolved LES simulation using turbulence recycling.

\section{THREE-DIMENSIONAL DIFFUSER FLOW}

Separated flow in a 3D rectangular diffuser has been studied in the experiments [11]. It was shown that the separation zone is strongly sensitive to geometric characteristics of the diffuser. This flow presents a challenge for RANS modeling approaches, RANS simulations of this flow have generally produced nonsatisfactory results [18]. The LES and hybrid RANS-LES studies were more successful in predicting flow behavior for this case [19]. However, when using synthetic turbulence, one usually obtains velocity and Reynolds stresses used to generate the synthetic velocity field from the RANS solution. Thus, unphysical velocity and stresses fields at the inflow can significantly worsen the results of the LES solution in the whole domain. To estimate the effect of inlet averaged velocity and Reynolds stresses on the LES solution, the simulations of the diffuser flow have been done with synthetic turbulence generated using RANS solution produced by $k-\omega$ SST model and EARSM-WJ-BSL model. Also, a LES run using recycling inflow generation method has been done.

The schematic of the diffuser is shown in Fig. 8. The Reynolds number based on bulk velocity $U_{b}$ in the inlet channel and height of the inlet channel $H$ is equal to $R e=10^{4}$. Flow in the inlet channel is assumed to be developed. Five simulation runs have been done for the flow: RANS simulations using $k-\omega$ SST

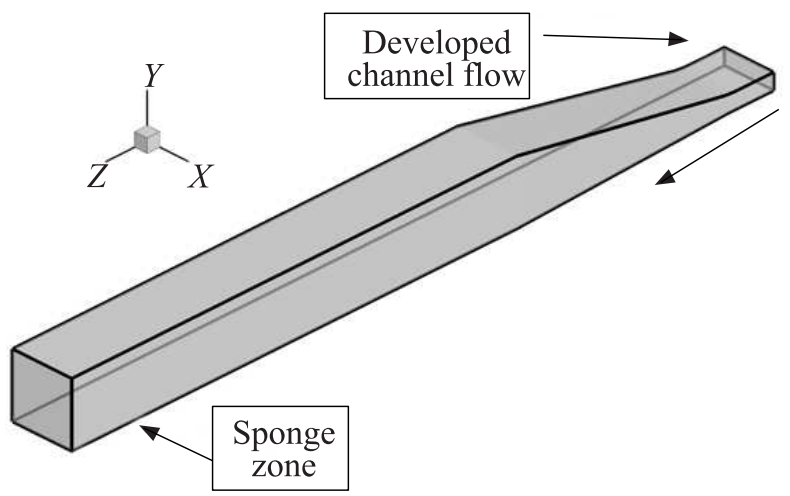

Figure 8 Schematic of the 3D diffuser 


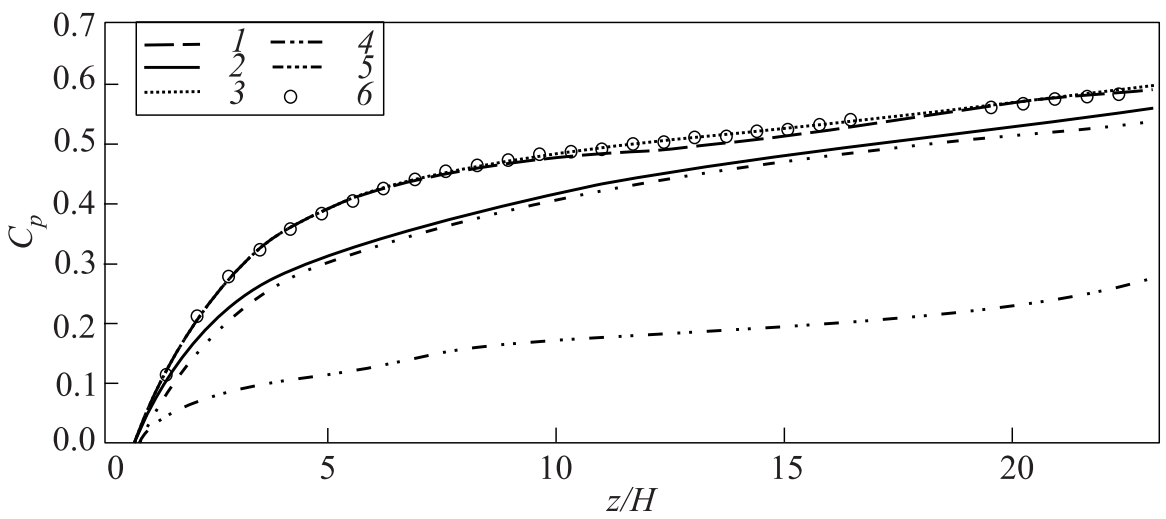

Figure 9 Comparison of pressure coefficient distribution at the lower wall of the diffuser: LES using turbulence recycling (1), using synthetic turbulence generated based on $k-\omega$ SST RANS (2) and EARSM-WJ-BSL RANS fields (3); RANS simulations using $k-\omega$ SST model (4) and EARSM-WJ-BSL model (5); and 6 - experiment

model and EARSM-WJ-BSL model, LES simulations using turbulence recycling and using synthetic turbulence generated based on $k-\omega$ SST RANS and EARSMWJ-BSL RANS fields.

Computational domain ranged from $x / H=-3$ to 55 for all the simulations except LES with recycling. For the recycling case, the inlet channel was extended to $x / H=-9$. The grid had dimensions $137 \times 77 \times 135$ for RANS simulations, $414 \times 77 \times 135$ for LES with synthetic turbulence, and $449 \times 77 \times 135$ for LES using recycling.

At the outlet boundary, constant pressure conditions have been used in RANS simulations. For LES simulation, a sponge zone with length $L / H=10$ has been used where the velocity and pressure fields were smoothly blended with RANS solution using cubic blending function. This was done to damp strong pressure waves reflecting from the outlet variable in unsteady simulation.

The comparison of the simulation results with experimental data [11] is shown in Figs. 9 and 10. The LES simulation using turbulence recycling produced results in excellent agreement with experimental data both for pressure distribution on lower wall (see Fig. 9) and averaged velocity fields (see Fig. 10). Thus, it is shown that LES using this grid and model produces good results for this flow.

The RANS results with $k-\omega$ SST model were in complete disagreement with experimental data while EARSM-WJ-BSL model predicted the pressure on the lower wall much better but still with differing significantly from the experimental results.

The results of LES runs with synthetic turbulence at the inlet boundary depend significantly on the averaged velocity and Reynolds stresses fields used to 


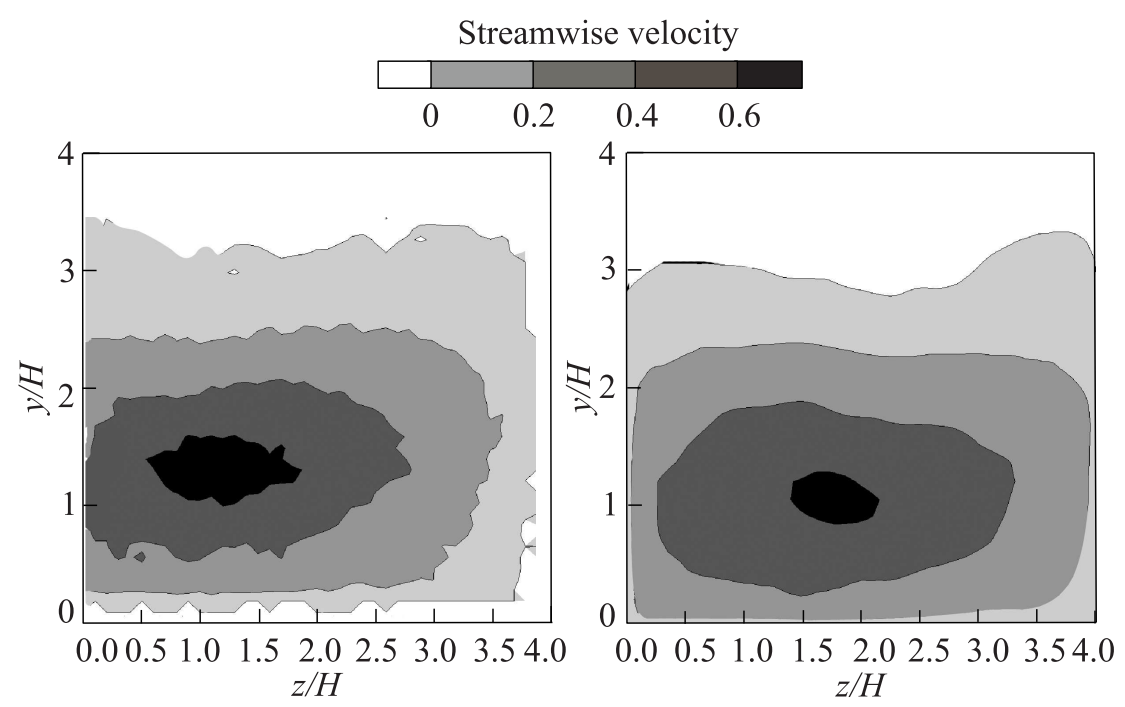

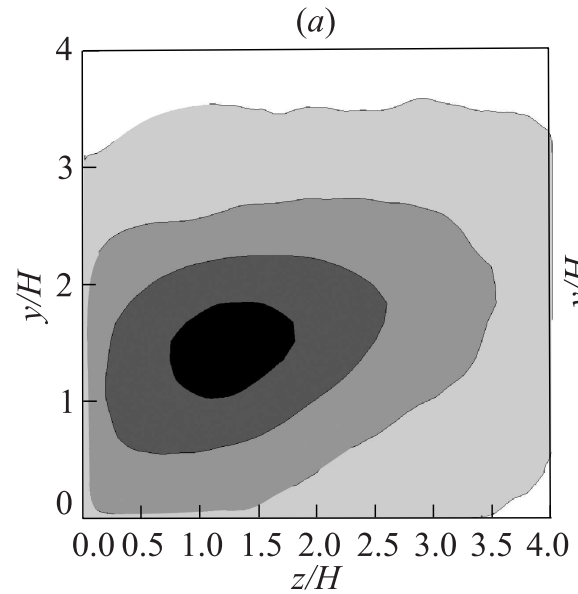

(c)

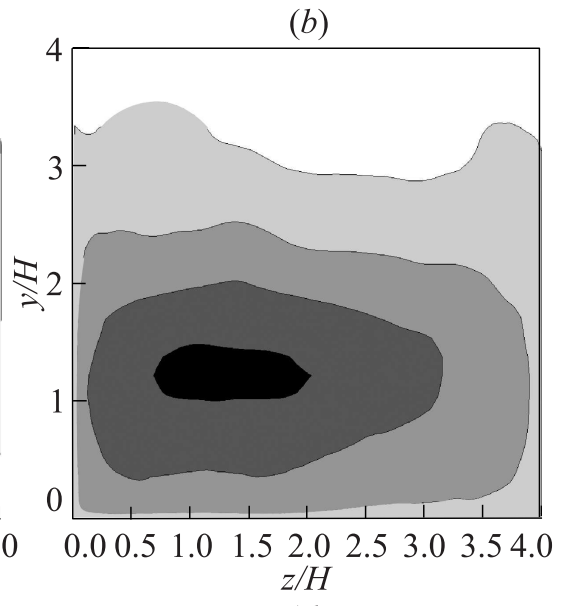

(d)

Figure 10 Comparison of averaged streamwise velocity fields at the exit plane of the diffuser $(x / H=15)$ : experiment $(a)$; LES using turbulence recycling $(b)$, using synthetic turbulence generated based on $k-\omega$ SST RANS (c); and EARSM-WJ-BSL RANS fields $(d)$

produce synthetic velocity fields. When RANS fields obtained with the EARSMWJ-BSL model were used as an input to the generator of synthetic turbulence, the results were in excellent agreement both with LES using recycling and with experimental data. The use of RANS fields produced by $k-\omega$ SST model to 
generate synthetic turbulent fluctuations significantly worsened the predictions of pressure and velocity fields.

\section{CONCLUDING REMARKS}

A recently developed method to generate synthetic turbulent velocity fluctuations has been applied to zonal RANS-LES simulations of complex turbulent flows including pressure-driven separation with downstream reattachment of the boundary layer and to a simulation of a complex 3D turbulent flow with secondary corner flows. It has been shown that zonal RANS-LES approach to simulation of turbulent flows provides the results in excellent agreements with experimental data for shallow the separation flows. Artificial turbulent content at the RANS-LES interface greatly improves prediction of the mean flow in the separation zone (compared to one of the most advanced existing hybrid RANSLES methods - IDDES) without significant degradation of the solution near the RANS-LES interface. For a turbulent flow in a 3D diffuser, it has been shown that the results depend strongly on the RANS solution used to create synthetic turbulent content at the inflow. When the inflow synthetic turbulence was created using the fields of velocity and turbulence variables produced with Reynolds-stress model EARSM-WJ-BSL [13] taken as the input, the results of the LES simulation agreed well with experimental data and with LES using recycling method. Noticeably worse results were obtained when using fields produced with linear eddy-viscosity model.

\section{ACKNOWLEDGMENTS}

This work was funded by the EU ATAAC (ACP8-GA-2009-233710) project, by Boeing Commercial Airplanes, and, partially, by the Russian Foundation for Basic Research (grant No. 09-08-00126).

\section{REFERENCES}

1. Lund, T. S., X. Wu, and K. D. Squires. 1998. Generation of turbulent inflow data for spatially-developing boundary layer simulations. J. Comput. Phys. 140:233-58.

2. Shur, M., P. R. Spalart, M. Strelets, and A. Travin. 2010. A rapid and accurate switch from RANS to LES in boundary layers using an overlap region. Flow Turb. Combust. 86:179-206.

3. Adamian, D., and A. Travin. 2010. An efficient generator of synthetic turbulence at RANS-LES interface in embedded LES of wall-bounded and free shear flows. 6th Conference (International) on Computational Fluid Dynamics Proceedings. 
4. Batten, P., U. Goldberg, and S. Chakravarthy. 2004. Interfacing statistical turbulence closures with large-eddy simulation. AIAA J. 42:485-92.

5. Kempf, A., M. Klein, and J. Janicka. 2005. Efficient generation of initial- and inflow-conditions for transient turbulent flows in arbitrary geometries. Flow Turb. Combust. 74:67-84.

6. Jarrin, N., S. Benhamadouche, D. Laurence, and R. Prosser. 2006. A syntheticeddy-method for generating inflow conditions for large-eddy simulations. Int. J. Heat Fluid Flow 27:585-93.

7. Greenblatt, D., K. B. Paschal, C.S. Yao, and J. Harris. 2005. A separation control CFD validation test case. Part 2. Zero efflux oscillatory blowing. 43rd AIAA Aerospace Sciences Meeting and Exhibit.

8. Rumsey, C. L., T. Gatski, W. L. Sellers, V. Vasta, and S. Viken. 2004. Summary of the 2004 CFD Validation Workshop on Synthetic Jets and Turbulent Separation Control. 2nd AIAA Flow Control Conference.

9. Blake, W.K. 1975. A statistical description of pressure and velocity fields at the trailing edges of a flat strut. David Taylor Naval Ship Research and Development Center. Technical Report 4241.

10. Leschziner, M. A., and F. Tessicini. 2009. Separated flow behind an aerofoil trailing edge without camber. In: DESider — a European effort on hybrid RANS-LES modelling. 207-16.

11. Cherry, E., C. Elkins, and J. Eaton. 2008 Geometric sensitivity of three-dimensional separated flows. Int. J. Heat Fluid Flow 29:803-11.

12. Menter, F. R. 1994. Two-equation eddy-viscosity turbulence models for engineering applications. AIAA J. 32:1598-605.

13. Menter, F. R., A. V. Garbaruk, and Y. Egorov. 2009. Explicit algebraic Reynolds stress models for anisotropic wall-bounded flows. 3rd European Conference for Aero-Space Sciences.

14. Shur, M., P.R. Spalart, M. Strelets, and A. Travin. 2008. A hybrid RANS-LES approach with delayed-DES and wall-modelled LES capabilities. Int. J. Heat Fluid Flow 29:1638-49.

15. Garbaruk, A., D. Magidov, M. Shur, M. Strelets, and A. Travin. 2006. Contribution by SPTU: Support of partners' efforts directed to implementation of DES technology. In: FLOMANIA - a European initiative on flow physics modelling. $101-8$.

16. Rogers, S.E., and D. Kwak. 1990. An upwind differencing scheme for the incompressible Navier-Stokes equations. AIAA J. 28:253-62.

17. Wang, M., and P. Moin. 2000. Computation of trailing-edge flow and noise using large-eddy simulation. AIAA J. 38:2201-9.

18. Karvinen, A., and H. Ahlstedt. 2008. Comparison of turbulence models in case of three-dimensional diffuser. Open Source CFD Conference (International).

19. Jakirlić, S., G. Kadavelil, M. Kornhaas, M. Schäfer, D. C. Sternel, and C. Tropea. 2010. Numerical and physical aspects in LES and hybrid LES/RANS of turbulent flow separation in a 3-D diffuser. Int. J. Heat Fluid Flow 31:820-32. 